\title{
CAPACIDAD Y VULNERABILIDAD DE LOS SUELOS DE LA COMUNIDAD VALENCIANA
}

\author{
Carlos Añó Vidal, Juan Sánchez Díaz, Carmen Antolín Tomás y Marta Goberna Estellés
} Centro de Investigaciones sobre Desertificación. C.S.I.C. - Universitat de València

\section{RESUMEN}

La infrautilización de la información que aporta la cartografía de suelos está ocasionada, entre otros motivos, por la presentación en términos exclusivamente científicos de los resultados generados por los reconocimientos edafológicos. En este trabajo, para facilitar el uso e interpretación de los mapas de suelos, se establecen las pautas generales de comportamiento de la mayoría de las unidades taxonómicas cartografiadas en la Comunidad Valenciana, indicando su capacidad y vulnerabilidad. El Indicador de Capacidad muestra la vocación intrínseca del medio edáfico y del entorno físico que permitirá o limitará el uso agrario. El Indicador de Vulnerabilidad considera las limitaciones potenciales, ocasionadas por una gestión deficiente, que pueden deteriorar las funciones del sistema edáfico.

Palabras clave: Mapas de suelos, indicador de capacidad, indicador de vulnerabilidad, evaluación de suelos, Comunidad Valenciana.

\section{ABSTRACT}

Pedological information represented in soil maps is offered solely in scientific terms. This is one of the reasons that give rise to its under-use. In this paper, we set the behaviour standards of the main taxonomic soil units mapped in the Valencia Region. The capability and vulnerability of these units are indicated in order to facilitate the understanding of the data provided by soil surveys. The Capability Index refers to the intrinsic vocation of both soil and its physical surroundings, and it determines land capability for farming use. The Vulnerability Index considers soil potential limitations occasioned by its mismanagement, which may lead to the decline of soil system functions.

Key words: Soil maps, capability index, vulnerability index, land evaluation, Valencia Region. 


\section{Introducción}

La cartografía de suelos aporta la caracterización, localización, distribución y clasificación de la tipología edáfica presente en un territorio. Esta información, imprescindible para evaluar la calidad del edafosistema, es también indispensable tanto en estudios de evaluación (Añó et al., 1997), planificación y ordenación territorial (Zinck, 1996) como de protección medioambiental (Indorante et al., 1996), ya que conocer las propiedades, la distribución, la extensión, la variabilidad espacial, la aptitud y vulnerabilidad del recurso edáfico es un requisito esencial para poder gestionar de manera sostenible el territorio (Dumanski, 1993; Bouma, 1994; McKenzie y McDonald, 1994; Basher, 1997).

Así, sería lógico que aumentara la demanda de estudios edáficos. Sin embargo, en las últimas dos décadas se ha cuestionado la utilidad de estos estudios (Ibáñez et al., 1993). Es más, la información de suelos con mucha frecuencia se desestima (Zinck, 1993). Entre las causas de esta situación, pueden destacarse las siguientes: presentación inadecuada de los resultados; ausencia de un sistema general de clasificación aceptado por toda la comunidad científica; pérdida parcial de información originado durante el proceso de elaboración de la cartografía, etc. (Dudal, 1987). Otros motivos que en España, durante muchos años, han restringido, o incluso rechazado, la utilización de la información de suelos en los estudios de planificación de usos del territorio, ha sido la escasez de mapas a escalas detalladas (Boixadera e Ibáñez, 1996), la ausencia de leyendas cartográficas que incorporasen la interpretación de los datos obtenidos por los reconocimientos edafológicos (Añó et al., 1998a) y, un aspecto que no es baladí, la utilización, en las memorias y leyendas de los mapas de suelos, de una terminología especializada, poco accesible a científicos procedentes de otras disciplinas.

Por tanto, acceder y, sobre todo, comprender la información derivada de los reconocimientos edafológicos es una de las labores previas a la asignación de los usos de un territorio, especialmente si consideramos que el suelo es un sistema complejo, abierto, dinámico, multifuncional y que carece de un comportamiento uniforme. Además, en el ámbito mediterráneo constituye un recurso no renovable a escala de tiempo humana en función de una tasa de renovación muy lenta. Por estos motivos las formaciones edáficas representan un elemento clave en la planificación y gestión de los recursos naturales.

Con el fin de ayudar a interpretar la información que aportan los mapas de suelos, a continuación establecemos las pautas generales de comportamiento de la mayoría de las unidades taxonómicas identificadas en la Comunidad Valenciana, indicando su capacidad y vulnerabilidad, conceptos más comprensibles que la denominación taxonómica. Actualmente los sistemas más utilizados para clasificar los suelos en España son la Soil Taxonomy (Soil Survey Staff, 1999) elaborada por el Servicio de Conservación de Suelos del Departamento de Agricultura de los Estados Unidos, y la leyenda del mapa de suelos del mundo propuesto por la Organización de las Naciones Unidas para la Agricultura y la Alimentación (FAO en su acrónimo inglés). La nomenclatura establecida por este último organismo internacional ha sido, y continúa siendo, el sistema de clasificación más usado para cartografiar los suelos en la Comunidad Valenciana. En este trabajo hemos considerado la versión de 1988 (FAO-UNESCO, 1988) que es la utilizada en los mapas de suelos realizados en el marco del proyecto LUCDEME y que, desde 1995, está publicando la Conselleria d'Agricultura de la Generalitat Valenciana. 


\section{Indicador de Capacidad y de Vulnerabilidad}

Los indicadores de capacidad y de vulnerabilidad constituyen la piedra angular de un método de evaluación de suelos diseñado en función de las peculiaridades biofísicas de la Comunidad Valenciana (Añó et al., 1998-1999). La evaluación de una serie de características intrínsecas y extrínsecas del medio edáfico, representadas en dieciséis parámetros, permite diagnosticar la capacidad del suelo y su vulnerabilidad a la degradación. En cada parámetro se ha establecido una gradación (Cuadros 1, 2 y 3). Los criterios utilizados en la gradación y los motivos que justificaron la elección de estos parámetros se encuentran desarrollados en Añó y Sánchez (1998) y Añó et al. (1998b).

El comportamiento del suelo y de los atributos del entorno físico considerados se plasma en el grado asignado a los distintos parámetros, que se agrupan en dos indicadores: capacidad y vulnerabilidad. Los parámetros disponibilidad de agua para las plantas, condiciones térmicas, pendiente, afloramientos rocosos, pedregosidad superficial o en la zona radicular, riesgo de inundación, espesor efectivo del suelo, hidromorfía, clase textural, propiedades químicas, alcalinidad, salinidad y erosión hídrica actual constituyen el Indicador de Capacidad que muestra la vocación intrínseca del medio edáfico y del entorno físico que permitirá o limitará el uso agrario. En función de los valores establecidos en cada uno de los factores, diferenciamos, a partir del grado máximo que pueden alcanzar los parámetros, entre Capacidad Muy Elevada, Elevada, Moderada, Baja y Muy Baja (Cuadro 4).

Los parámetros riesgo de erosión hídrica potencial, riesgo de salinización o alcalinización y vulnerabilidad del suelo a la contaminación configuran el Indicador de Vulnerabilidad que señala las limitaciones potenciales, ocasionadas por prácticas inadecuadas o una gestión deficiente, que pueden deteriorar las múltiples funciones que pueden desarrollar los suelos. De acuerdo al grado establecido en los tres parámetros diferenciamos entre Vulnerabilidad Baja, Moderada y Alta (Cuadro 5). El análisis completo de los dos indicadores puede consultarse en Añó (1996).

\section{Evaluación de los suelos de la Comunidad Valenciana}

En esta primera aproximación a la evaluación de las unidades y subunidades edáficas que reflejan los mapas de suelos más recientes de la Comunidad Valenciana, se ha excluido la evaluación de los Alisoles, Acrisoles e Histosoles por varios motivos: las dos primeras unidades presentan unas características químicas, complejo de cambio, que no aparecen bien diferenciadas en el sistema FAO-UNESCO de 1988. Además, son suelos integrantes de ecosistemas silicícolas típicos, aunque puntuales, de nuestro ámbito. Por su parte, los Histosoles están íntimamente relacionados con los Gleysoles, unidades que analizamos posteriormente. También, por razones bien diferentes, se excluye la evaluación de los Antrosoles, suelos con unas propiedades determinadas por las actividades antrópicas que han modificado intensamente, incluso enterrado, los horizontes edáficos originales. En el Cuadro 6 se resume la capacidad y la vulnerabilidad de los suelos que se han evaluado.

\subsection{Fluvisoles}

Los suelos de fondo de valle y de las zonas aluviales tienen una amplia representación en el ámbito mediterráneo, ocupando las llanuras litorales y las primeras terrazas de los cursos fluviales en las áreas interiores. Estas unidades taxonómicas se localizan sobre pen- 

Cuadro 2

\begin{tabular}{|c|c|c|c|c|c|c|c|}
\hline \multicolumn{8}{|c|}{$\begin{array}{c}\text { Cuadro } 2 \\
\text { GRADACIÓN DE LOS PARÁMETROS ESPESOR EFECTIVO, SALINIDAD, ALCALINIDAD, CLASE TEXTURAL, HIDROMORFÍA, } \\
\text { PROPIEDADES QUÍMICAS Y RIESGO DE INUNDACIÓN }\end{array}$} \\
\hline \multicolumn{2}{|c|}{ ESPESOR EFECTIVO (X) } & \multicolumn{6}{|c|}{ CLASE TEXTURAL (T) } \\
\hline X1. Profundo & $>90 \mathrm{~cm}$ & \multirow{2}{*}{\multicolumn{2}{|c|}{$\begin{array}{l}\text { T1. Franco-arcillosa } \\
\text { T2. Franco-arcillo-limosa } \\
\text { Franco-arcillo-arenosa }\end{array}$}} & \multicolumn{2}{|c|}{ T4. Arcillosa } & \multicolumn{2}{|c|}{ T7. Franco-arenosa } \\
\hline X2. Mod. Profundo & $50-90 \mathrm{~cm}$ & & & \multicolumn{2}{|c|}{ T5. Franco-limosa } & \multicolumn{2}{|c|}{ T8. Limosa } \\
\hline X3. Somero & $30-50 \mathrm{~cm}$ & \multirow{2}{*}{\multicolumn{2}{|c|}{$\begin{array}{l}\text { T3. Arcillo-limosa } \\
\text { Arcillo-arenosa }\end{array}$}} & \multirow{2}{*}{\multicolumn{2}{|c|}{ T6. Franca }} & \multirow{2}{*}{\multicolumn{2}{|c|}{ T9. Arenosa }} \\
\hline X4. Poco profundo & $10-30 \mathrm{~cm}$ & & & & & & \\
\hline X5. Muy poco profundo & $<10 \mathrm{~cm}$ & \multirow{5}{*}{\multicolumn{6}{|c|}{$\begin{array}{l}\text { HIDROMORFÍA (H) } \\
\text { H1. Suelos bien drenados. Síntomas de hidromorfismo a una profundidad superior a } \\
50 \mathrm{~cm} \text { desde la superficie. } \\
\text { H2. Suelos moderadamente bien drenados. Síntomas de hidromorfismo a una } \\
\text { profundidad entre } 30 \text { y } 50 \mathrm{~cm} \text { desde la superficie. } \\
\text { H3. Suelos imperfectamente drenados. Síntomas de hidromorfismo a una profundidad } \\
\text { inferior a } 30 \mathrm{~cm} \text { desde la superficie. }\end{array}$}} \\
\hline \multicolumn{2}{|c|}{ SALINIDAD (S) } & & & & & & \\
\hline S1. Muy baja & $<2 \mathrm{dS} / \mathrm{m}$ & & & & & & \\
\hline S2. Baja & $2-4 \mathrm{dS} / \mathrm{m}$ & & & & & & \\
\hline S3. Moderada & $4-8 \mathrm{dS} / \mathrm{m}$ & & & & & & \\
\hline S4. Alta & $8-16 \mathrm{dS} / \mathrm{m}$ & \multicolumn{6}{|c|}{ PROPIEDADES QUÍMICAS (Q) } \\
\hline \multirow[t]{2}{*}{ S5. Muy alta } & $>16 \mathrm{dS} / \mathrm{m}$ & & $\begin{array}{l}\text { MO } \\
(\%)\end{array}$ & $\begin{array}{c}\mathrm{CaCO}_{3} \\
(\%)\end{array}$ & $\begin{array}{c}\text { Caliza } \\
\text { activa }(\%)\end{array}$ & $\begin{array}{c}\mathrm{CIC} \\
(\mathrm{cmol}+/ \mathrm{Kg})\end{array}$ & pH $\left(1: 2.5 \mathrm{H}_{2} \mathrm{O}\right)$ \\
\hline & & Q1. Muy adecuadas & $>2$ & $10-20$ & $<5$ & $>20$ & $6.1-7.8$ \\
\hline \multicolumn{2}{|c|}{ ALCALINIDAD (N) (RAS) } & Q2. Adecuadas & $1-2$ & $20-30$ & $5-10$ & $10-20$ & $5.5-6.1 / 7.8-8.5$ \\
\hline S1. Muy baja & $<5 \%$ & Q3. Inadecuadas & $<1$ & $30-50$ & $10-15$ & $<10$ & $<5.5 />8.5$ \\
\hline S2. Baja & $5-8 \%$ & \multirow{4}{*}{\multicolumn{6}{|c|}{$\begin{array}{l}\text { RIESGO DE INUNDACIÓN (I) } \\
\text { I1. Bajo. Frecuencia de inundaciones en periodos de tiempo superiores a } 25 \text { años. } \\
\text { I2. Medio. Frecuencia de inundaciones en periodos de tiempo de } 10 \text { a } 25 \text { años. } \\
\text { I3. Alto. Frecuencia de inundaciones en periodos de tiempo inferiores a } 10 \text { años. }\end{array}$}} \\
\hline S3. Moderada & $8-11 \%$ & & & & & & \\
\hline S4. Alta & $11-15 \%$ & & & & & & \\
\hline S5. Muy alta & $>15 \%$ & & & & & & \\
\hline
\end{tabular}



Cuadro 4

INDICADOR DE CAPACIDAD

\begin{tabular}{|c||l|}
\hline \multicolumn{1}{|c|}{ INDICADOR DE CAPACIDAD } \\
\hline \hline ELE ADA & $\begin{array}{l}\text { Los parámetros nunca superan el grado 1, a excepción de la clase textural, las } \\
\text { propiedades químicas, la pedregosidad y el riesgo de inundación que pueden } \\
\text { alcanzar el grado 2. }\end{array}$ \\
\hline \multirow{2}{*}{ ELE ADA } & $\begin{array}{l}\text { Los parámetros nunca superan el grado 2, a excepción de la clase textural, la } \\
\text { pedregosidad y el riesgo de inundación que pueden alcanzar el grado 3 }\end{array}$ \\
\hline \multirow{3}{*}{ MODERADA } & $\begin{array}{l}\text { Los parámetros no superan el grado 3, excepto la pedregosidad y la clase } \\
\text { textural que pueden alcanzar, respectivamente, el grado 4 y el 7. }\end{array}$ \\
\hline \multirow{2}{*}{ BAJA } & $\begin{array}{l}\text { El grado de los parámetros que determinan el Indicador (pendiente, espesor } \\
\text { efectivo, afloramientos rocosos, pedregosidad, erosión hídrica actual, } \\
\text { salinidad, alcalinidad y clase textural arenosa) oscilan entre 3 y 4, excepto la } \\
\text { granulometría y la pendiente que pueden alcanzar, respectivamente, el grado } \\
9 \text { y 5. Los valores establecidos en el resto de parámetros no participan en la } \\
\text { caracterización del Indicador }\end{array}$ \\
\hline MUY BAJA & $\begin{array}{l}\text { Los parámetros que determinan el Indicador (pendiente, espesor, } \\
\text { afloramientos rocosos, pedregosidad, erosión hídrica actual, salinidad, } \\
\text { alcalinidad y clase textural arenosa) alcanzan o superan, en un número } \\
\text { superior a dos, el grado 5. Los valores establecidos en el resto de parámetros } \\
\text { no intervienen en la caracterización del Indicador. }\end{array}$ \\
\hline
\end{tabular}

Cuadro 5

INDICADOR DE VULNERABILIDAD

\begin{tabular}{|c||l|}
\hline \multicolumn{2}{|c|}{ INDICADOR DE ULNERABILIDAD } \\
\hline \hline \multirow{3}{*}{ BAJA } & $\begin{array}{l}\text { El riesgo de salinización o alcalinización (RS1-RN1), la vulnerabilidad de los } \\
\text { suelos a la contaminación (V1) y/o el riesgo de erosión potencial (Ep1/2) es } \\
\text { bajo. }\end{array}$ \\
\hline \multirow{3}{*}{ MODERADA } & $\begin{array}{l}\text { El riesgo de salinización o alcalinización (RS2-RN2), la vulnerabilidad de los } \\
\text { suelos a la contaminación (V2) y/o el riesgo de erosión potencial (Ep3/4) es } \\
\text { moderado. }\end{array}$ \\
\hline \multirow{2}{*}{ ELE ADA } & $\begin{array}{l}\text { El riesgo de salinización o alcalinización (RS3-RN3), la vulnerabilidad de los } \\
\text { suelos a la contaminación (V3) y/o el riesgo de erosión potencial (Ep5/6) es } \\
\text { alto o muy alto. }\end{array}$ \\
\hline
\end{tabular}


Cuadro 6

SÍNTESIS DE CAPACIDAD Y VULNERABILIDAD DE LOS SUELOS DE LA COMUNIDAD VALENCIANA

\begin{tabular}{|c|c|c|c|}
\hline ORDEN DE SUELO & SUBUNIDAD & $\begin{array}{l}\text { INDICADOR DE } \\
\text { CAPACIDAD }\end{array}$ & $\begin{array}{l}\text { INDICADOR DE } \\
\text { ULNERABILIDAD }\end{array}$ \\
\hline \multirow[t]{2}{*}{ FLU ISOLES } & Eútrico/Calcárico/Dístrico & \multirow{2}{*}{$\begin{array}{l}\text { MUY ELEVADA } \\
\text { ELEVADA }\end{array}$} & $\begin{array}{c}\text { BAJA } \\
\text { MODERADA }\end{array}$ \\
\hline & Sálico & & ALTA \\
\hline GLEYSOLES & $\begin{array}{c}\text { Cálcicos/Móllicos } \\
\text { Tiónicos } \\
\end{array}$ & MODERADA & MODERADA \\
\hline REGOSOLES & $\begin{array}{l}\text { (Hiper) Gypsífero } \\
\text { (Hiper) Calcárico } \\
\text { Eútrico/Úmbrico }\end{array}$ & $\begin{array}{l}\text { MODERADA } \\
\text { BAJA } \\
\text { MUY BAJA }\end{array}$ & ALTA \\
\hline LEPTOSOLES & $\begin{array}{l}\text { Rendzínico/Móllico/Úm- } \\
\text { brico/Eútrico/Dístrico }\end{array}$ & MUY BAJA & ALTA \\
\hline \multirow[t]{2}{*}{ ARENOSOLES } & $\begin{array}{c}\text { Álbico/Gleico } \\
\text { Háplico/Calcárico }\end{array}$ & BAJA & \multirow[t]{2}{*}{ ALTA } \\
\hline & Cámbico & MUY BAJA & \\
\hline \multirow{5}{*}{ CAMBISOLES } & Eútrico & $\begin{array}{c}\text { ELEVADA } \\
\text { MODERADA }\end{array}$ & MODERADA \\
\hline & \multirow{2}{*}{ Crómico } & $\begin{array}{c}\text { BAJA } \\
\text { MUY BAJA }\end{array}$ & ALTA \\
\hline & & $\begin{array}{c}\text { ELEVADA } \\
\text { MODERADA }\end{array}$ & MODERADA \\
\hline & Gleico & $\begin{array}{l}\text { MODERADA } \\
\text { BAJA } \\
\text { MUY BAJA } \\
\end{array}$ & ALTA \\
\hline & Calcárico & $\begin{array}{l}\text { ELEVADA } \\
\text { MODERADA }\end{array}$ & MODERADA \\
\hline \multirow{4}{*}{ CALCISOLES } & Háplico & $\begin{array}{c}\text { ELEVADA } \\
\text { MODERADA } \\
\text { BAJA } \\
\end{array}$ & $\begin{array}{c}\text { BAJA } \\
\text { MODERADA } \\
\text { ALTA } \\
\end{array}$ \\
\hline & Pétrico & MUY VARIABLE & \\
\hline & \multirow{2}{*}{ Lúvico } & $\begin{array}{c}\text { BAJA } \\
\text { MUY BAJA }\end{array}$ & \multirow{2}{*}{ ALTA } \\
\hline & & $\begin{array}{c}\text { ELEVADA } \\
\text { MODERADA }\end{array}$ & \\
\hline SOLONCHAKS & $\begin{array}{c}\text { Cálcico } \\
\text { Gleico } \\
\end{array}$ & $\begin{array}{c}\text { BAJA } \\
\text { MUY BAJA }\end{array}$ & ALTA \\
\hline \multirow{2}{*}{ KASTANO EMS } & $\begin{array}{l}\text { Háplico } \\
\text { Lúvico }\end{array}$ & \multirow{2}{*}{ BAJA } & ALTA \\
\hline & Cálcico & & $\begin{array}{l}\text { MODERADA } \\
\text { ALTA } \\
\end{array}$ \\
\hline CHERNO EMS & Cálcico & BAJA & $\begin{array}{l}\text { MODERADA } \\
\text { ALTA } \\
\end{array}$ \\
\hline \multirow{2}{*}{ PHAEO EMS } & Lúvico & MODERADA & $\begin{array}{c}\text { BAJA } \\
\text { MODERADA } \\
\text { ALTA } \\
\end{array}$ \\
\hline & $\begin{array}{l}\text { Calcárico } \\
\text { Háplico }\end{array}$ & $\begin{array}{l}\text { MODERADA } \\
\text { BAJA }\end{array}$ & $\begin{array}{l}\text { MODERADA } \\
\text { ALTA }\end{array}$ \\
\hline LU ISOLES & $\begin{array}{l}\text { Háplico } \\
\text { Crómico } \\
\text { Álbico } \\
\text { Cálcico }\end{array}$ & $\begin{array}{l}\text { MUY ELEVADA } \\
\text { ELEVADA } \\
\text { MODERADA } \\
\text { MUY BAJA }\end{array}$ & $\begin{array}{l}\text { MODERADA } \\
\text { ALTA }\end{array}$ \\
\hline
\end{tabular}


dientes llanas, en todos los casos sin superar el 3\%. En sectores, sobre todo interiores, pueden desarrollarse sobre pendientes acondicionadas por bancales que no obstaculizan la mecanización. La amplia representación territorial determina que las condiciones térmicas y la disponibilidad de recursos hídricos para las plantas no sean homogéneas, abarcando todos los grados establecidos. En última instancia, los valores asignados a estos dos parámetros condicionarán el Indicador de Capacidad.

Estos suelos, poco evolucionados edáficamente, están bien drenados y su desarrollo a partir de depósitos aluviales recientes se traduce en un espesor efectivo que no dificulta el enraizamiento. Existe una ausencia generalizada de afloramientos rocosos, mientras la pedregosidad superficial o en la zona radicular es muy variable, en muchas ocasiones con porcentajes que no superan el $15 \%$, valores que pueden aumentar aunque predominando las gravas. La clase textural es variable por su carácter poligenético mientras las propiedades químicas son adecuadas, presentando un bajo contenido en materia orgánica $(<2 \%)$, un contenido variable en carbonatos, baja capacidad de intercambio catiónico y pH que oscila entre 5,5 y 8,5. Por tanto son parámetros que, a pesar de no alcanzar valores muy positivos, nunca incidirán negativamente en la evaluación.

El riesgo de inundación es medio o alto, en función de la proximidad de la unidad a los cursos fluviales y de las obras de infraestructura realizadas en los últimos años relacionadas con la canalización de los ríos (v.gr. Segura) o modificación del cauce (v.gr. Turia). El grado de erosión hídrica actual y el riesgo potencial es bajo, con ausencia de morfología erosiva excepto la laminar. La vulnerabilidad a la contaminación es baja o moderada, mientras la salinidad actual es variable dependiendo del tipo de material de origen y de la calidad de las aguas de riego. Este último factor, unido al tipo de drenaje interno, provoca que algunas unidades taxonómicas tengan un riesgo de salinización moderado o alto.

Las subunidades identificadas en la Comunidad Valenciana (Fluvisoles eútricos, Fluvisoles calcáricos, Fluvisoles dístricos y Fluvisoles sálicos) al margen de su diferenciación taxonómica presentan una Capacidad Muy Elevada o Elevada y una Vulnerabilidad Baja o Moderada, excepto la última subunidad con idéntico Indicador de Capacidad pero con Vulnerabilidad Alta consecuencia del riesgo de salinización.

\subsection{Gleysoles}

Suelos localizados en depresiones mal drenadas y en zonas de marjales próximas al litoral que no reciben aportes regulares de sedimentos. El horizonte superficial puede tener unas características y un comportamiento similar a los suelos aeróbicos, mientras que en los primeros $50 \mathrm{~cm}$ de profundidad aparecen condiciones reductoras por la presencia de una capa de agua que satura el espacio poroso, al menos en alguna época del año. En conjunto, el hidromorfismo, que genera un drenaje imperfecto, es el parámetro más importante que condiciona totalmente el desarrollo edáfico y sus posibilidades de utilización.

Estas unidades, ubicadas en todos los casos sobre pendientes que no superan el 3\%, presentan una alta, incluso excesiva para muchas especies vegetales, disponibilidad de agua para las plantas y, en un gran número de ocasiones, unas condiciones térmicas muy adecuadas. El material de origen, no consolidado y nunca de textura gruesa, condiciona un espesor efectivo del suelo superior a $90 \mathrm{~cm}$. En la mayoría de las ocasiones predomina la ausencia de afloramientos rocosos y una escasa pedregosidad superficial. La clase textural dominante es arcillosa y limosa, mientras las propiedades químicas son inadecuadas. Por su parte, la salinidad actual es variable, el riesgo de inundación es medio o alto y el grado de erosión 
hídrica actual y el riesgo de erosión potencial es bajo. Por el contrario, el riesgo de salinización o alcalinización es moderado y la vulnerabilidad a la contaminación es baja o moderada.

En conjunto son suelos con una Capacidad Moderada y una Vulnerabilidad Moderada. Las diferencias taxonómicas que permiten identificar las subunidades presentes en las tierras de la Comunidad Valenciana (Gleysoles cálcicos, Gleysoles móllicos y Gleysoles tiónicos) no modifican los Indicadores establecidos.

\subsection{Regosoles}

Suelos con muy escaso desarrollo genético formados a partir de materiales no consolidados, excluyendo a los de textura gruesa o que presentan propiedades flúvicas. Sus características (a excepción de un mayor o menor grado de incorporación de materia orgánica en el horizonte superficial) están directamente relacionadas con el material geológico, por tanto con escasa incidencia de los procesos edafogenéticos. La evolución está limitada por los procesos de erosión y/o por las características físicas y químicas del material de origen que provocan una gran variabilidad tanto en sus propiedades como en su posible utilización.

En general estos suelos, presentes en todos los ambientes climáticos de la Comunidad Valenciana, se desarrollan sobre pendientes variables, normalmente superiores al 15\% (P4 a P6). La disposición topográfica favorece un buen drenaje, salvo en materiales impermeables (H2), y la ausencia de riesgo de inundación. Las características del material de origen confieren a esta unidad un elevado espesor efectivo (X1-X2), con escasos o nulos afloramientos rocosos, presentando, sólo en algunas ocasiones, una pedregosidad frecuente o numerosa cuando el sustrato es de origen coluvial. Por tanto, estas características, en general, permiten un enraizamiento profundo. Por otro lado, la alcalinidad y salinidad actual es baja o moderada, presentando, sobre todo en los Regosoles gypsíferos, un riesgo de salinización o alcalinización moderado o alto. La vulnerabilidad a la contaminación es moderada o alta y, sobre pendientes acentuadas, el grado de erosión hídrica actual y el riesgo de erosión potencial es alto o muy alto, con una morfología erosiva muy variada.

Las diferencias entre las distintas subunidades residen, sobre todo, en las propiedades químicas y texturales, aspectos que no modificarán el Indicador de Capacidad o Vulnerabilidad. Los Regosoles calcáricos, desarrollados sobre margas más o menos arenosas, tienen una textura muy variable (T1 a T8) y una propiedades químicas inadecuadas que reflejan el bajo porcentaje de materia orgánica y su mayor o menor contenido en carbonato. Cuando este anión supera el $50 \%$ puede establecerse, a nivel de fase, el carácter hipercalcárico que frena la evolución del perfil. Los Regosoles gypsíferos, desarrollados a partir de yesos o margas yesíferas, presentan textura fina (T1 a T4) y propiedades químicas inadecuadas marcadas por la preponderancia del anión sulfato que puede determinar, a nivel de fase, el carácter hipergypsífero motivando alta salinidad y/o alcalinidad. Los Regosoles eútricos suelen desarrollarse sobre areniscas o arcillas, ambas descarbonatadas. La presencia de bases en el suelo garantiza unas propiedades químicas más adecuadas. Por último, los Regosoles úmbricos tienen un elevado porcentaje de materia orgánica y un bajo contenido en bases por un material de origen pobre en ellas que provoca unas características químicas desfavorables, predominado las texturas francas. En conjunto estos suelos presentan una Capacidad Moderada, Baja o Muy Baja y una Vulnerabilidad Alta. 


\subsection{Leptosoles}

Los suelos que presentan esta denominación, desarrollados la mayoría de las veces sobre materiales calcáreos consolidados (calizas y dolomías) y, en menor medida, afloramientos de areniscas, están determinados por el parámetro espesor efectivo, menor de $30 \mathrm{~cm}$, que condiciona un escaso desarrollo del perfil y confiere al suelo una reducida disponibilidad para el enraizamiento. Estas unidades tienen una amplia representación en todos los ambientes montañosos de la Comunidad con unas condiciones térmicas y de disponibilidad hídrica poco homogéneas. Están localizados sobre zonas de topografía abrupta (v.gr. escarpes o cuestas) o más suave (altiplanos o páramos). Los Leptosoles localizados en algunas zonas forestales pueden ser el resultado de un largo proceso de evolución hasta alcanzar, aunque frágiles, las condiciones de estabilidad. Sin embargo, por regla general, su presencia no está relacionada directamente con ningún factor formador, pues en la mayoría de las ocasiones son la consecuencia de la denudación total del suelo preexistente.

Las subunidades identificadas en la Comunidad Valenciana son Leptosoles rendzínicos, Leptosoles móllicos, Leptosoles úmbricos, Leptosoles eútricos, Leptosoles dístricos y Leptosoles líticos. Las tres primeras subunidades poseen unas propiedades físicas y químicas que pueden ser adecuadas o muy adecuadas: alto contenido en materia orgánica y elevada estabilidad estructural, aspectos que favorecen la infiltración. En el resto de subunidades estas propiedades son menos favorables. Independientemente de las diferencias taxonómicas, las limitaciones actuales y potenciales son muy severas (sobre todo en la última subunidad) condicionando el Indicador de Capacidad y de Vulnerabilidad: pendientes, preferentemente, abruptas; afloramientos rocosos abundantes o dominantes; pedregosidad abundante y espesor efectivo del suelo poco profundo o, en los Leptosoles líticos, muy poco profundo. Por su parte el grado de erosión hídrica actual es bajo o ligero en los Leptosoles rendzínicos, móllicos y úmbricos, y ligero o moderado en los Leptosoles eútricos y dístricos, mientras el riesgo de erosión potencial es, en todos los casos, alto o muy alto. Por definición los Leptosoles líticos están en fase lítica. En conjunto todas las subunidades tienen una Capacidad Muy Baja y una Vulnerabilidad Alta.

\subsection{Arenosoles}

Suelos no evolucionados, por tanto con un perfil prácticamente indiferenciado, que tienen una textura más gruesa que franco arenosa hasta una profundidad de $100 \mathrm{~cm}$. Con unas condiciones térmicas ( $\mathrm{C} 1$ a $\mathrm{C} 3)$ y disponibilidad de agua (Dh1 a Dh3) variable, su origen está determinado, en las áreas interiores, por la litología y, en los sectores litorales, por la geomorfología. Estos suelos son de textura gruesa, cualidad que influye directamente en las desfavorables características físicas y químicas de los mismos: baja capacidad de retención de agua, baja estabilidad estructural, escaso contenido en materia orgánica y reducida capacidad de cambio. Por tanto, las características, sobre todo granulométricas, determinan en estas unidades una Capacidad Baja o Muy Baja.

Entre las subunidades cartografiadas en la Comunidad Valenciana, los Arenosoles álbicos desarrollados sobre areniscas del Buntsandstein están asociados en ámbitos continentales a Leptosoles, mientras en el litoral forman el cordón dunar, muchas veces asociados a Arenosoles gleicos, subunidad que muestra propiedades hidromórficas (H1 a H3). En los dos casos están localizados sobre pendientes suaves, poseen un moderado o alto espesor efectivo, con ausencia de afloramientos rocosos, pedregosidad, salinidad o riesgo de inun- 
dación. Las características físicas y químicas confieren una vulnerabilidad a la contaminación alta, mientras el grado de erosión hídrica actual es moderado y el riesgo potencial es moderado o alto. En conjunto los dos suelos tienen una Capacidad Baja y una Vulnerabilidad Alta, presentando un frágil equilibrio en el ecosistema.

Los Arenosoles háplicos y los Arenosoles calcáricos, a diferencia de las anteriores subunidades, se desarrollan sobre pendientes superiores (P3-P4) y presentan unos porcentajes más elevados de afloramientos rocosos (R2-R3) y de pedregosidad (G3). Además, el riesgo de erosión potencial es alto o muy alto. Por tanto, ambos suelos poseen una Capacidad Baja y una Vulnerabilidad Alta. Por último, los Arenosoles cámbicos, formados a partir de areniscas del Buntsandstein, se localizan en pendientes mayores (P4-P5) y tienen unas características más desfavorables que determinarán el Indicador de Capacidad, manteniendo el mismo Indicador de Vulnerabilidad: baja disponibilidad para el enraizamiento, numerosos afloramientos rocosos (R4-R5) y una pedregosidad muy abundante (G4-G5). En este caso la Capacidad es Muy Baja, manteniéndose una Vulnerabilidad Alta.

\subsection{Cambisoles}

Suelos poco evolucionados con un horizonte B cámbico con evidencias de alteración que permiten diferenciarlo del material de origen. Las muestras de alteración responden a procesos muy diferentes: eliminación de carbonatos, estructura moderadamente desarrollada, cierta movilización de arcillas, alteración y liberación de óxidos de hierro, etc. Estos rasgos definen suelos formados sobre materiales geológicos variados, presentes en un gran número de ambientes climáticos de la Comunidad Valenciana. La ausencia de características homogéneas dificulta la valoración conjunta de la unidad taxonómica, siendo necesaria su evaluación por subunidades.

Los Cambisoles eútricos, suelos con horizonte superficial de escasa incorporación de materia orgánica, evidencian alteración subsuperficial que modifica su estructura de roca y están carentes de óxidos de hierro deshidratados y de carbonato cálcico entre, por lo menos, 20 y $50 \mathrm{~cm}$. En la Comunidad Valenciana su formación suele ser a partir de materiales poco carbonatados, presentando un espesor efectivo moderadamente profundo y una clase textural, en general, franca. Los afloramientos rocosos no constituyen una limitación ni al desarrollo radicular ni al laboreo, ya que el material de origen se fractura fácilmente en cantos heterométricos frecuentes o numerosos. Están localizados en áreas de suaves a moderadas pendientes, a veces abancaladas, y están presentes en ambientes con condiciones térmicas moderadamente adecuadas y alta disponibilidad de agua para las plantas, sin presentar rasgos hidromórficos ni riesgo de inundación. Las propiedades químicas son favorables, con ausencia de salinidad o alcalinidad. A su vez, las características químicas, en gran parte, condicionarán una vulnerabilidad a la contaminación moderada. El grado de erosión hídrica actual oscila de bajo a moderado mientras el riesgo potencial es moderado o acusado. En conjunto son suelos con una Capacidad Elevada o Moderada y una Vulnerabilidad Moderada.

Los Cambisoles crómicos son suelos no calcáreos que manifiestan su moderada alteración tanto en la ausencia de estructura de roca como en la segregación y liberación de óxidos deshidratados de hierro que confieren al horizonte subsuperficial un color pardo fuerte a rojo o más rojo. La existencia de estas subunidades en la Comunidad Valenciana obedece a mecanismos bien diferenciados en función del material de origen del que proceden. Por un lado, a partir de una roca madre coherente y dura (v.gr. calizas y/o dolomías más o menos 
fisuradas) se desarrollan suelos, en general, decapitados, localizados en las cimas de los relieves (P1 a P3). En líneas generales la disponibilidad de agua para las plantas es baja y los afloramientos rocosos son numerosos que impiden un desarrollo horizontal continuo del horizonte cámbico. Esta característica genera suelos de escaso espesor efectivo (X3-X4) con presencia de abundantes cantos angulosos muy poco rodados, más o menos gruesos, superficiales y/o en el perfil (G3-G4) provocando, a nivel de fase, un carácter rúpticolítico. Estas subunidades están bien drenadas, no tienen riesgo de inundación y carecen de propiedades salinas y/o alcalinas. Estos suelos, morfológicamente muy semejantes a los Luvisoles crómicos en fase rúptico-lítica excepto su textura menos arcillosa (T1 a T3, T5 y T6), pueden, en función de su nivel de degradación, aparecer en fase lítica. La Capacidad es Baja o Muy Baja, mientras el riesgo alto o muy alto de erosión potencial determinan una Vulnerabilidad Alta.

Por el contrario, los Cambisoles crómicos, desarrollados a partir de materiales menos consolidados pobres en carbonato cálcico (v.gr. arcillas, areniscas o argilitas), muestran una gran semejanza con los Cambisoles eútricos, excepto una localización sobre pendientes más variadas (P1 a P3) y una clase textural que puede oscilar entre rangos más amplios (T4 a T7), aspectos que no modifican el valor establecido en los Indicadores: Capacidad Moderada o Elevada y Vulnerabilidad Moderada.

Los Cambisoles gleicos, emparentados genéticamente con los Gleysoles, ocupan zonas topográficas llanas. La disposición topográfica y el predominio de la granulometría limosa condicionan un encharcamiento ocasional ( $\mathrm{H} 1 \mathrm{a} \mathrm{H} 3)$ que provoca, en los primeros $100 \mathrm{~cm}$ a partir de la superficie, la aparición de procesos de oxidación-reducción. Son suelos con un elevado espesor efectivo, sin afloramientos rocosos, escasa pedregosidad (G1-G2) y un grado de erosión hídrica actual y riesgo potencial bajo. Por el contrario, las condiciones químicas pueden ser, por los procesos red-ox, desfavorables (Q2-Q3), el riesgo de inundación es alto, la vulnerabilidad a la contaminación es moderada y la salinidad o alcalinidad puede ser elevada o muy elevada igual que el riesgo de salinización o alcalinización. En conjunto son suelos con una Capacidad Moderada, Baja o Muy Baja y una Vulnerabilidad Alta.

Los Cambisoles calcáricos se desarrollan frecuentemente a partir de materiales aluviales o coluviales más o menos carbonatados. Ubicados sobre pendientes suaves, presentan un elevado espesor efectivo, ausencia de afloramientos rocosos y, en función del tipo de depósito original, una pedregosidad muy variable (G1 a G4) que, incluso en los grados más elevados, no suele constituir una grave limitación al uso de maquinaria agrícola. La clase textural también varía (T1 a T7) en función de la granulometría del depósito. El drenaje es adecuado, el riesgo de inundación bajo o moderado, las propiedades químicas son adecuadas y la salinidad o alcalinidad baja o moderada. La vulnerabilidad a la contaminación es moderada, el grado de erosión hídrica actual y el riesgo potencial es bajo o ligero. Por regla general, estos suelos tienen una Capacidad Elevada o Moderada y una Vulnerabilidad Moderada.

\subsection{Calcisoles}

En la génesis del suelo las condiciones semiáridas favorecen la formación de horizontes con enriquecimiento secundario de carbonato cálcico que caracteriza a los Calcisoles. Estas unidades, muy bien representadas en la Comunidad Valenciana, están presentes tanto en la llanura litoral como en las zonas montañosas del interior prácticamente sobre cualquier fisiografía, predominando el lavado y acumulación de carbonato asociado a procesos 
geomorfológicos de formación de glacis, conos de deyección, abanico aluvial, etc. Estas áreas, relativamente llanas y a menudo abancaladas, reciben aguas bicarbonatadas que, en función de las características topográficas y el balance hídrico negativo, precipitan en forma de caliza pulverulenta blanda, nódulos más o menos irregulares (Calcisoles háplicos) o, incluso, costras (Calcisoles pétricos) que pueden limitar seriamente la profundidad del perfil.

Los Calcisoles háplicos tienen una amplia distribución en el ámbito mediterráneo. En la franja costera se desarrollan sobre pendientes que no superan el 8\%, disposición topográfica que es variable en las zonas del interior. Están asociados a procesos que han permitido el lavado de carbonato cálcico en las partes superiores del perfil formando un horizonte Bw de eliminación de carbonato, acumulando este material de forma discreta, sin constituir una limitación en el espesor del suelo (X1-X2). Por regla general, la disponibilidad de agua para las plantas (Dh1 a Dh3) y las condiciones térmicas $(\mathrm{C} 1$ a $\mathrm{C} 3)$ son variables, los afloramientos rocosos son escasos, la pedregosidad no supera el $40 \%$ y las propiedades químicas pueden ser inadecuadas. La granulometría, aunque variable, suele ser bastante equilibrada, el drenaje es adecuado, la salinidad y alcalinidad es baja igual que la vulnerabilidad a la contaminación. El grado de erosión hídrica actual y el riesgo de erosión potencial varía en función de las condiciones locales. Estas características pueden configurar suelos muy productivos, frecuentemente dedicados a cítricos, con un riesgo de inundación medio y un riesgo de salinización o alcalinización bajo o moderado. En general tienen una Capacidad Elevada o Moderada y una Vulnerabilidad Baja o Moderada. En sectores, sobre todo del interior, pueden presentar una Capacidad Baja y una Vulnerabilidad Alta.

Los Calcisoles pétricos tienen un horizonte de carbonato cálcico endurecido que puede limitar (en función de su consistencia, continuidad y/o profundidad) el desarrollo radicular de las plantas y su disponibilidad de agua y nutrientes. Las diferencias respecto a la anterior subunidad son el espesor efectivo, parámetro muy variable (X1 a X4) dependiendo de la profundidad de la costra, y el porcentaje de pedregosidad que varía (G1 a G6) según el estado de conservación de la misma ya que en muchos casos ha sido levantada manteniendo sus restos en el perfil. Los valores concretos establecidos en los dos parámetros determinarán el Indicador de Capacidad que es muy variable.

Las variaciones climáticas de las últimas etapas geológicas han propiciado la aparición de suelos en los que predomina la acumulación o lavado de ciertos elementos en función de las características pluviométricas de cada periodo. Algunos Luvisoles muestran actualmente una tendencia a la recarbonatación secundaria de su horizonte árgico que va destruyendo la estructura poliédrica del mismo a medida que se acumula el carbonato cálcico en formas esferoidales blandas, nodulares o costras, terrificando progresivamente el horizonte Bt. Las propiedades de los Calcisoles lúvicos son semejantes a las de los Luvisoles de los que proceden. A partir de sustratos consolidados, sin desarrollo continuo del horizonte árgico recarbonatado, la Capacidad es Baja o Muy Baja y la Vulnerabilidad Alta. Por el contrario, sobre sustratos menos cohesionados la Capacidad es Elevada o Moderada y la Vulnerabilidad Alta.

\subsection{Solonchaks}

Esta denominación engloba, por definición, suelos salinos típicos de depresiones litorales o interiores. En la Comunidad Valenciana están ubicados, por regla general, en el ámbito costero y presentan una capa freática superficial de aguas salinas o salobres que provocan 
una elevada concentración de sales solubles que ascienden por capilaridad, acumulándose, incluso, en la superficie del suelo. Esta característica impide el desarrollo de la mayoría de las especies vegetales, permitiendo únicamente la implantación de comunidades especializadas y configurando ecosistemas singulares (saladares).

La mayoría de los parámetros que configuran el Indicador de Capacidad y de Vulnerabilidad son muy favorables (espesor efectivo elevado, ausencia de rocosidad o pedregosidad, condiciones térmicas muy adecuadas, grado de erosión hídrica actual y riesgo potencial bajo, etc.) pero la elevada salinidad (S4-S5), unas propiedades químicas inadecuadas y un riesgo de salinización alto caracterizan suelos con una Capacidad Baja o Muy Baja y Vulnerabilidad Alta, independientemente de las subunidades identificadas en la Comunidad Valenciana (Solonchak cálcico-gleico y Solonchak gleico-gípsico) que sólo indican la composición de otras sales acumuladas (carbonato cálcico o yeso) y el tipo de drenaje.

\subsection{Suelos isohúmicos}

Esta denominación engloba un conjunto de suelos forestales (Kastanozems, Chernozems y Phaeozems) que reflejan unas condiciones térmicas (moderadamente adecuadas o poco adecuadas) y de disponibilidad hídrica (alta o moderada) específicas y un estado de equilibrio con la vegetación, traducido en una incorporación mayor y más profunda de materia orgánica. La característica común es la presencia de un horizonte móllico que proporciona una estructura construida muy desarrollada y un porcentaje de agregados estables muy alto. Esta elevada estabilidad estructural confiere al suelo una alta permeabilidad e infiltración y, por tanto, muy baja escorrentía.

Los Kastanozems son de ámbitos más cálidos, con menor contenido en materia orgánica y estabilidad estructural. Al nivel de subunidad, los Kastanozems háplicos, por regla general asociados a Leptosoles rendzínicos y Leptosoles móllicos, están situados sobre coluvios calizos en pendientes muy abruptas, presentando un espesor efectivo somero, abundante pedregosidad, escasos afloramientos rocosos, buen drenaje, muy baja salinidad y una clase textural variable (T1 a T7). Por su parte, la vulnerabilidad a la contaminación es moderada y el grado de erosión hídrica actual oscila entre bajo y moderado, mientras el riesgo de erosión potencial es alto o muy alto. Por tanto, estos suelos tienen una Capacidad Baja y una Vulnerabilidad Alta.

Los Kastanozems cálcicos se desarrollan en partes bajas de las laderas y muestran la formación de un horizonte cálcico, generalmente no cementado, fruto de la acumulación de caliza pulverulenta. Respecto a la anterior subunidad están localizados sobre pendientes inferiores, pueden presentar menor disponibilidad para el enraizamiento (X3-X4) y el riesgo de erosión potencial oscila entre moderado y alto. En conjunto, son suelos con una Capacidad Baja y una Vulnerabilidad Moderada o Alta.

Por último, los Kastanozems lúvicos, suelos localizados en pendientes inferiores al $25 \%$, tienen mejores propiedades granulométricas (T1 a T4) y químicas aunque el porcentaje de afloramientos rocosos es superior (R4) y el espesor efectivo es menor (X4), características que determinan una Capacidad Baja. Por su parte, la Vulnerabilidad, en función de un riesgo de erosión potencial moderado o acusado, es Moderada

Entre los suelos isohúmicos, los Chernozems tienen una menor distribución territorial en la Comunidad Valenciana motivado, probablemente, por las exigencias de color que restringen su identificación taxonómica. En gran medida, la abundancia de materiales carbonatados impide una mayor humificación y melanización de estos suelos desarrollados en 
unas condiciones térmicas (C3) e hídricas (Dh1) idénticas que los Phaeozems. La única subunidad cartografiada (Chernozems cálcicos) presenta unas características similares a las descritas en los anteriores suelos, mostrando una Capacidad Baja y, dependiendo del riesgo de erosión potencial, una Vulnerabilidad Moderada o Alta.

Los Phaeozems, suelos con una presencia puntual aunque de gran interés biogeográfico, tienen un desarrollo mediatizado por el factor climático o el material de origen, sobre todo sustratos semiconsolidados y permeables que permitan el lavado y eliminación de los carbonatos. En líneas generales son unidades con muy baja salinidad, ausencia de propiedades hidromórficas, propiedades químicas muy adecuadas, baja disponibilidad para el enraizamiento, numerosa pedregosidad superficial, escasos o frecuentes afloramientos rocosos, baja vulnerabilidad a la contaminación y un grado de erosión hídrica actual que oscila de bajo a moderado.

A nivel de subunidad los Phaeozems lúvicos tienen una clase textural, por regla general, arcillosa, están ubicados sobre pendientes inferiores al $15 \%$ y muestran un riesgo de erosión potencial ligero, moderado o acusado. Estos suelos presentan una Capacidad Moderada y una Vulnerabilidad Baja, Moderada o Alta en zonas con pendientes acentuadas donde el riesgo de erosión potencial es elevado (Ep5-Ep6). Por su parte, los Phaeozems calcáricos y los Phaeozems háplicos son subunidades con una clase textural variable (T1 a T7), están localizados sobre pendientes superiores al 15\% (P3 a P5) y evidencian un riesgo de erosión potencial que abarca de ligero a muy alto. Así, la Capacidad es Moderada o Baja y la Vulnerabilidad Moderada o Alta.

\subsection{Luvisoles}

Estos suelos son ricos en nutrientes y presentan un horizonte de acumulación de arcilla. En condiciones ambientales mediterráneas la existencia de un horizonte B árgico subsuperficial suele asociarse a materiales diversos. Los Luvisoles desarrollados a partir de materiales consolidados (calizas o dolomías) se encuentran, por regla general, en disposición topográfica de altiplano o en pendientes que oscilan entre 3 y $25 \%$, asociados a procesos kársticos de disolución de las rocas carbonatadas. Son unidades con una amplia distribución territorial, representados tanto en ambientes litorales como continentales, que determina una gran variabilidad en las condiciones térmicas $(\mathrm{C} 1$ a $\mathrm{C} 3)$ y en la disponibilidad de agua para las plantas (Dh1 a Dh3).

El suelo, generalmente, presenta un perfil truncado, por tanto el horizonte de acumulación de arcilla con mucha frecuencia alcanza la superficie desarrollado en forma de bolsadas, rellenando oquedades y grietas o fisuras de las rocas sin continuidad lateral. Esta característica limita el espesor efectivo del suelo (X3-X4) y provoca la presencia de abundantes afloramientos rocosos (R4 a R6). Además, los suelos, por regla general, están cubiertos por un pavimento pedregoso que mantiene la humedad y permite, bajo una vegetación sobre todo arbustiva, el desarrollo de un pequeño horizonte de acumulación de materia orgánica. El drenaje, por la naturaleza kárstica fisurada del material de origen, suele ser rápido. En ocasiones excepcionales, en los Luvisoles desarrollados sobre argilitas o materiales areniscosos en áreas con precipitaciones elevadas, las arcillas pueden desferrificarse parcialmente observándose procesos red-ox producto de una cierta hidromorfía $(\mathrm{H} 2-\mathrm{H} 3)$, consecuencia de la impermeabilidad de las arcillas al desflocularse. Esta circunstancia origina un tipo de subunidad (Luvisol gleico) con una presencia puntual en la Comunidad Valenciana que únicamente puede cartografiarse a escalas detalladas. 
La textura del horizonte argílico es fina (T1 a T4), sin embargo puede variar en la superficie en función de la presencia de horizontes álbicos que, en algunas ocasiones, originan una clase textural arenosa (T7 o T9). Las propiedades químicas son, en general, muy adecuadas si se desarrollan bajo vegetación natural, ya que junto a un elevado porcentaje de materia orgánica, la capacidad de intercambio catiónico es alta (debido más a las cargas variables de la materia orgánica que al tipo de arcilla que suele ser de moderada actividad), los contenidos de carbonatos y caliza activa son bajos, y los valores de $\mathrm{pH}$ están próximos a la neutralidad. Por su parte, la alcalinidad y la salinidad actual es muy baja. Estas unidades, por su ubicación topográfica, no presentan riesgos de inundación. Además, la vulnerabilidad a la contaminación es baja y no existe riesgo de salinización o alcalinización. Por último, el grado de erosión hídrica actual es ligero con predominio absoluto de la erosión laminar, mientras el riesgo de erosión potencial puede llegar a ser acusado o alto (Ep4-Ep5).

En conjunto, son suelos con una Capacidad Muy Baja y una Vulnerabilidad Moderada o Alta. Sin embargo, en zonas con pendientes moderadas (8-15\%), con desarrollo horizontal del suelo y escasos afloramientos rocosos, por tanto con mayor espesor del horizonte superficial, con textura equilibrada y menor riesgo de erosión potencial, presentan una Capacidad Moderada o Baja y una Vulnerabilidad Baja o Moderada.

Los Luvisoles desarrollados a partir de materiales menos consolidados (arcillas, argilitas o, incluso, areniscas) tienen una menor distribución territorial en el ámbito mediterráneo valenciano. En estos suelos, a diferencia del caso anterior, el horizonte producto de la iluviación de la arcilla aparece continuo, a mayor o menor profundidad, determinando un espesor efectivo del suelo, al menos, moderado (X1 a X3). Otro rasgo característico en estos Luvisoles es la ausencia de afloramientos rocosos y de pedregosidad. En algunas ocasiones permanece el horizonte eluvial provocando un cambio textural abrupto entre ambos horizontes (de arenoso - T9 - a mucho más fino - T1 a T4-) que modifica bruscamente las condiciones hídricas de la unidad. Esta situación puede provocar, en disposiciones topográficas llanas, la aparición de un cierto grado de hidromorfía (H2) en la zona de contacto entre ambos horizontes, mostrando síntomas de asfixia radicular. El resto de parámetros muestran unas condiciones semejantes a los Luvisoles descritos anteriormente. Por tanto, esta unidad taxonómica presenta una Vulnerabilidad Moderada, mientras la Capacidad es Muy Elevada, Elevada o Moderada.

Las subunidades, con suficiente entidad cartográfica, presentes en la Comunidad Valenciana son Luvisoles háplicos, Luvisoles crómicos, Luvisoles álbicos y Luvisoles cálcicos. Las diferencias taxonómicas en este segundo nivel no implican, necesariamente, una modificación en su evaluación.

\section{Conclusiones}

Los mapas de suelos y sus correspondientes memorias tradicionalmente se han presentado en términos exclusivamente científicos, sin aportar ningún otro tipo de indicación. Este aspecto ha impedido a un gran número de posibles usuarios comprender la información edáfica. El comportamiento de los distintos suelos distribuidos en un territorio varía en función de las condiciones medioambientales y de las características socioeconómicas específicas de cada zona. La posibilidad, con una fuerte carga determinista, de establecer una relación directa entre los tipos de suelos con su capacidad y vulnerabilidad es difícil de aplicar a un espacio tan poco homogéneo como el valenciano. Sin embargo, sí es posible establecer unos valores medios que reflejen su comportamiento general. Así, con el objetivo de 
facilitar la interpretación de la cartografía edafológica hemos evaluado, mediante los indicadores de capacidad y de vulnerabilidad, la mayoría de las unidades taxonómicas descritas en la Comunidad Valenciana.

Por regla general, los usuarios que solicitan información edáfica más que el conocimiento de los detalles taxonómicos de los suelos identificados en una zona, demandan interpretaciones prácticas de los mapas de suelos. Por tanto, habría que generar un tipo de cartografía que aportara información útil para la evaluación y planificación del territorio. Desde este punto de vista, los mapas de suelos que se efectuaron en el marco del Plan Nacional de Cartografía Temática Ambiental incorporaron, en la leyenda cartográfica, el indicador de capacidad y el de vulnerabilidad. De este modo, en los 11 mapas que se realizaron a diferentes escalas durante la fase piloto (1996-97) las unidades cartográficas, que sintetizan tanto las características edáficas como las de su entorno biofísico, se establecieron basándose en la tipología del suelo y en los dos indicadores.

En última instancia, el uso final del suelo está condicionado por factores de diferente naturaleza: políticos, culturales, económicos etc. Pero, el conocimiento de la capacidad y vulnerabilidad del sistema edáfico aporta un tipo de información fundamental para poder planificar el desarrollo equilibrado y sostenible de las actividades productivas y, por tanto, asignar adecuadamente los usos del territorio; sobre todo si consideramos que la utilización inapropiada del suelo, recurso escaso y no renovable a corto y medio plazo, puede provocar su degradación de manera irreversible, especialmente en condiciones ambientales mediterráneas que determinan una deficiente capacidad de regeneración o recuperación de los suelos alterados por las actividades humanas.

\section{Referencias}

AÑó, C. (1996): Metodología de evaluación de suelos para el ámbito mediterráneo. Tesis Doctoral, Facultad de Geografía e Historia, Universitat de València.

AÑÓ, C. y SÁNCHEZ, J. (1998): Land use capability methodology for Mediterranean regions. Applications to land use planning. En: A. RODRÍGUEZ, C.C. JIMÉNEZ y M.L. TEJEDOR (eds.), The soil as a strategic resource: degradation processes and conservation measures, Geoforma Ediciones, Logroño, pp. 459-471.

AÑÓ, C.; SÁNCHEZ, J. y ANTOLÍN, C. (1997): Análisis y valoración de los sistemas de evaluación de suelos en España. Evolución, tendencias actuales y perspectivas futuras. Estudios Geográficos, 228, pp. 331-353.

AÑÓ, C.; SÁNCHEZ, J. y ANTOLÍN, C. (1998a): Interpretación de la información edafológica en el ámbito mediterráneo valenciano: Indicador de capacidad e indicador de vulnerabilidad. Edafología, 4, pp. 117-130.

AÑÓ, C.; SÁNCHEZ, J. y ANTOLÍN, C. (1998b): Evaluación del potencial edáfico en el País Valenciano. Cuadernos de Geografía, 63, pp. 3-16.

AÑÓ, C.; SÁNCHEZ, J. y ANTOLÍN, C. (1998-1999): Orientaciones de uso agrario: una metodología para la planificación de usos del suelo en el ámbito mediterráneo. Boletín de la Real Sociedad Geográfica, 134-135, pp. 187-209.

BASHER, L.R. (1997): Is pedology dead and buried? Australian Journal of Soil Research, 35, pp. 979-994.

BOIXADERA, J. e IBÁÑEZ, J.J. (1996): Spain. Soil survey and soil databases. En: C. LE BAS \& M. JAMAGNE (Eds.), Soil databases to support sustainable development, INRA-SESCPF, Joint Research Centre-IRSA, Orleans, pp. 99-105. 
BOUMA, J. (1994): Sustainable land use as a future focus for pedology. Soil Science Society of America Journal, 58, pp. 645-646.

DUDAL, R. (1987): The role of pedology in meeting the increasing demands on soils. Soil Survey and Land Evaluation, 7 (2), pp. 101-110.

DUMANSKI, J. (1993): Strategies and opportunities for soil survey information and research. ITC Journal, 1993-1, pp. 36-41.

FAO-UNESCO (1988): Soil Map of the World. Revised Legend. World Soil Resources Report 60, FAO, Roma, 119 pp.

IBÁÑEZ, J.J.; ZINCK, A.J. y JIMÉNEZ, R. (1993): Soil survey: old and new challenges. ITC Journal, 1993-1, pp. 7-14.

INDORANTE, S.J., McLEESE, R.L.; HAMMER, R.D.; THOMPSON, B.W. y ALEXANDER, D.L. (1996): Positioning soil survey for the 21st century. Journal of Soil and Water Conservation, 51 (1), pp. 21-28.

McKENZIE, N.J. y McDONALD, W. (1994): Developments in Australian land resource assessment. Australian Journal of Soil and Water Conservation, 7, pp. 19-22.

SOIL SURVEY STAFF (1999): Soil Taxonomy. A Basic System of Soil Classification for Making and Interpreting Soil Surveys. United States Department of Agriculture, Natural Resources Conservation Service, Washington DC, 869 pp.

ZINCK, J.A. (1993): Introduction. ITC Journal, 1993-1, pp. 2-6.

ZINCK, J.A. (1996): La información edáfica en la planificación del uso de las tierras y el ordenamiento territorial. En: J. AGUILAR, A. MARTÍNEZ \& A. ROCA (Eds.), Evaluación y manejo de suelos, Junta de Andalucía, Granada, pp. 49-75. 